\title{
Divergence in emergence time of host races due to differential gene flow
}

\author{
R. K. Butlin
}

School of Pure and Applied Biology, University of Wales College of Cardiff, PO Box 915, Cardiff CF1 3TL, U.K.

Differential gene flow between adjacent plant populations, induced by environmental influences on reproductive characters such as flowering time, may increase isolation between them (Stam, 1983). With the aid of a simulation model, an analogous process is demonstrated for populations of phytophagous insects. Allochronic isolation due to differences in host plant phenology can be accentuated in sympatry. Interpretations of divergence between host-associated insect populations should take account of the potential effects of differential gene flow before invoking explanations involving selection.

\section{INTRODUCTION}

The evolutionary origin of intrinsic barriers to gene flow is a major area of current controversy. One particular point at issue is the role of selection in the evolution of pre-mating isolation between divergent populations in either parapatric or sympatric contexts (Paterson, 1985; Butlin, 1989; Diehl and Bush, 1989). This debate has tended to concentrate on the relative importance of selection for increased assortative mating resulting from the production of hybrids with reduced fitness, reinforcement, on the one hand and divergence in allopatry on the other hand, which may be due to drift or selection pressures not directly related to isolation. Stam (1983) made an important contribution to this controversy by demonstrating that differential gene flow may contribute to divergence in flowering time between closely adjacent plant populations. This can occur in the absence of selection and can contribute to reproductive isolation between the two populations.

The mechanism proposed by Stam (1983) operates as follows. Two adjacent plant populations occupy distinct habitats, such as mining areas and nearby unpolluted soils (McNeilly and Antonovics, 1968). In each population there is genetic variation for flowering time but in one habitat the mean flowering time is delayed due to environmental conditions. In the habitat in which flowering is delayed, pollination by plants in the adjacent habitat is most likely to be by individuals that are genetically late flowering. Thus alleles for late flowering will flow selectively into the population whose flowering is delayed by environmental effects. The converse will be true for the early flowering population which will receive an excess of early flowering alleles from the adjacent population. Stam (1983) modelled this process and demonstrated that it can produce substantial divergence in flowering time and thus a marked reduction in gene flow between the two populations. This in turn can facilitate adaptation of the two populations to their local environmental conditions.

The potential significance of differential gene flow had been demonstrated previously in an experimental investigation by Thoday and Gibson (1970) but it does not seem to be widely appreciated. In this note I wish to point out that differential gene flow may accentuate phenological differences between insect populations feeding on different host plants. This clearly has importance in the context of models of sympatric speciation in phytophagous insects in which allochronic isolation is often considered a major barrier to gene flow (Bush, 1975).

\section{EMERGENCE TIME MODEL}

Consider an insect population that utilizes two host species, A and B. Adult emergence is delayed 
on host $\mathrm{B}$ relative to host $\mathrm{A}$ either because $\mathrm{B}$ is a less suitable host or, as in the Enchenopa binotata complex (Wood and Guttman, 1982), because hatching is cued to host phenology and this is delayed in B. Within the population as a whole genetic variation for adult emergence time exists, reflecting underlying variation in hatch time, development rate or maturation time. The situation is then directly analogous to that modelled by Stam (1983) except that there is no geographic component. If female dispersal is limited such that a female emerging on $\mathrm{A}$ has a greater than random probability of laying her eggs on a host of species $\mathrm{A}$, as is also true for Enchenopa binotata (Wood and Guttman, 1982) and probably many phytophagous insects, then this can replace the geographical separation. Females developing on A will tend to mate with males from $B$ with genetically early emergence and will tend to lay their eggs on A hosts. Thus the insect population on A will evolve towards early emergence and that on B towards later emergence. This will occur as a result of preferential gene flow without the need to implicate either selection directly on emergence time or selection for isolation resulting from adaptation to the alternate host plants. The divergence in emergence time will generate assortative mating along host plant lines.

I have constructed a simple simulation model to confirm this argument. A population of 500 individuals is divided, initially equally, between the two host types. Emergence times of individuals are drawn at random from a normal distribution with phenotypic variance $V$. Mean emergence time is set arbitrarily at 100 days on host $A$ and is delayed by $d$ days on host B. Insects survive for time $s$ days from emergence and males emerge (or mature) $s / 2$ days before females. (Without this protandry early emerging males have an overall advantage and the whole population evolves rapidly towards earlier development.) On emergence each female samples males at random until she finds a mate that is alive and sexually mature when she mates. If the female samples half the male population without finding a mate she is aged one day and the sampling is restarted. Females mate only once and may fail to mate before dying at age $s$ days. This mating scheme implies that male dispersal is at random with respect to host and great enough to ensure that males do not have

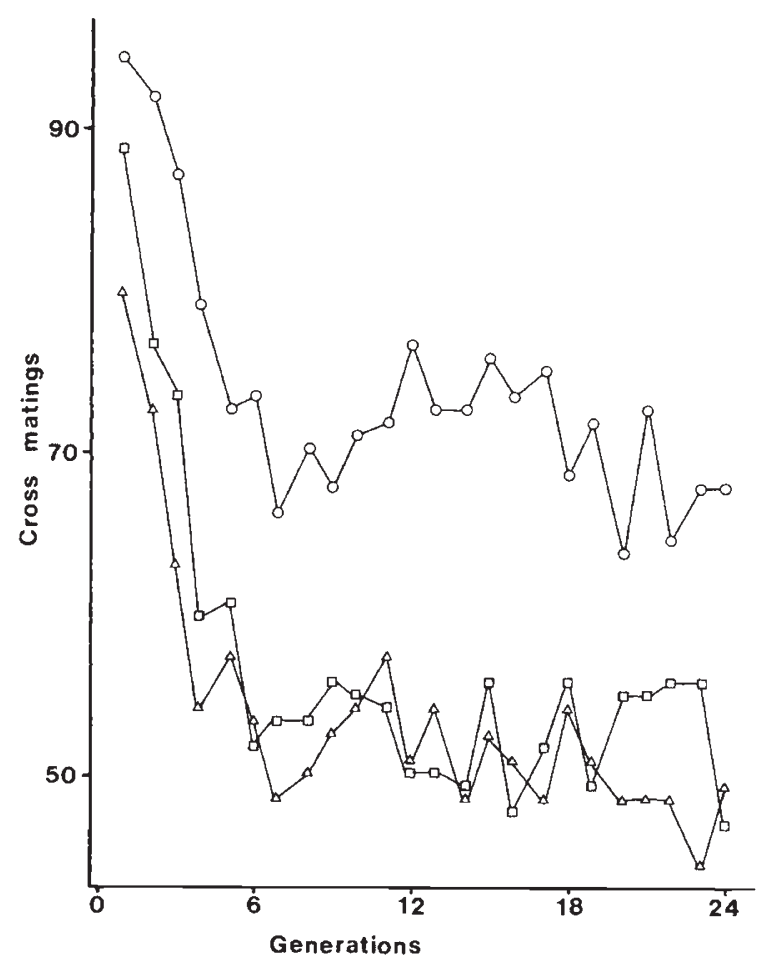

Figure 1 Mean values for five runs of the simulation for (a) the difference in emergence time between the two hosts and (b) the number of matings between individuals from different hosts. Parameters: $d=10, V=50, h^{2}=0.9, a=0.99, s=5$ (triangles), 10 (squares), 20 (circles). Random mating expectation for number of cross matings $=125$. 
an elevated chance of mating with females on the plant on which they developed. Mated females lay their eggs in one batch on the host on which they developed with probability $a$ and on the alternate host with probability 1-a. This could be due to active oviposition preference or simply to limited dispersal. The heritability of emergence time, $h^{2}$, and the phenotypic variance, $V$ (excluding the effect of host), are assumed to remain constant. The phenotypic values of the offspring are drawn from a normal distribution with mean $h^{2}$. (midparent value) and variance $\left(1-0 \cdot 5 h^{4}\right) . V$ (Bulmer, 1980) to which is added the effect of the host on which they are laid.

The simulation has been run for a variety of combinations of the parameters, $d, s, a, h^{2}$, and $V$, and for five replicate runs of thirty generations in each case. The results (figs 1 and 2) confirm the potential for the evolution of partial reproductive isolation by this mechanism. Most of the divergence in emergence time occurs in the first five to ten generations (fig. 1(a)) and is accompanied by a reduction in the number of matings between individuals derived from different hosts (fig. 1(b)). The various parameters have readily understandable effects on the magnitude of this reduction (fig. 2) which parallel those observed by Stam (1983).

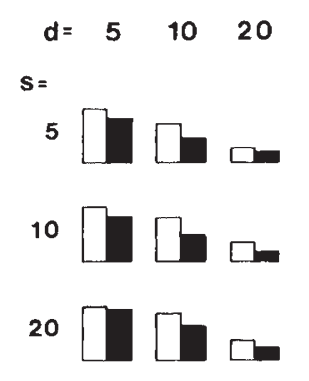

A

$$
5
$$

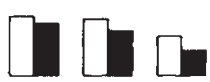

10

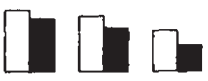

20

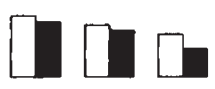

C

Figure 2 Effect of parameter settings on the reduction in number of cross matings. Open bars $=$ number of cross matings in generation 1 , closed bars = mean number of cross matings in generations 26-30 (mean of five simulation runs in each case). Parameters: $\mathrm{A}-V=50, h^{2}=0.9, a=$ $0.99 . \mathrm{B}-V=50, h^{2}=0.9, a=0.90 . \mathrm{C}-V=200, h^{2}=0.9$, $a=0.99$. D $-V=200, h^{2}=0.5, a=0.99$.
Variation in the environmental effect, $d$, interacts with the survival, $s$, such that where survival time is long and $d$ is small the influence of differential migration is small because mating is nearly random. The reduction in cross mating is greatest for intermediate values of both parameters but where survival is short and $d$ is large there is little gene flow initially and therefore little extra divergence. The phenotypic variance influences divergence in a way similar to survival time: where $V$ is large both the initial gene flow and the ultimate divergence are increased, particularly for large $d$. These parameters have parallels with flowering duration, pollen dispersal, and the environmental effect of habitat on flowering time in Stam's model. Divergence in emergence time and reduction in cross mating are greater for higher heritability and female host preference (or lower female dispersal), a.

In an alternative version of the model in which emergence time was influenced by two alleles at a single locus, allele frequencies showed the expected divergence. In simulation runs with $d=10$, $s=10, a=0.9$, with substitution of an A for an $a$ allele adding 10 days to the emergence time, and with environmental standard deviation of 9 days the mean allele frequency difference between host associated populations in generation 30 was $0 \cdot 31$ ( 5 runs). Initial allele frequency was 0.5 on each host. This corresponds to a mean emergence time difference of $16 \cdot 2$ days and a drop in cross matings to a mean of $61 \cdot 2$.

\section{DISCUSSION}

These results clearly indicate, as Stam (1983) suggested, that the mechanism of differential gene flow can produce divergence between sub-populations in a variety of settings and for a range of characters and, therefore, deserves to be given wider consideration. In the context of host associated divergence in phytophagous insects the process is potentially important in two ways. Firstly, demonstrations of genetic differentiation in emergence time between populations derived from alternative hosts may be accounted for by this mechanism. They cannot be taken as evidence for either adaptation to the phenology of the host or selection to increase allochronic isolation unless the biassed gene flow explanation can be discounted. Thus the genetic component of differentiation in emergence times between apple and hawthorn derived Rhagoletis pomonella (Smith, 1988) does not necessarily imply 
the existence of host races adapted to their respective hosts.

On the other hand, the mechanism of differential gene flow may contribute to divergence between host associated populations. The isolation that can be produced by this process alone is self limited since divergence depends on gene flow. However, the accentuation of isolation produced initially by environmental effects can facilitate adaptation as Stam (1983) demonstrated explicitly. This adaptation could take the form of further divergence in phenology, host preferences, or other adaptations to exploit the particular features of the hosts which might reduce the fecundity of individuals that migrate between hosts or the survival of their offspring. Any one of these would tend to decrease further the gene flow between host associated populations, without any involvement of "reinforcement" (Butlin, 1989), and together they may be sufficient to lead to speciation.

The Enchenopa binotata complex provides a well studied example in which differential gene flow could have contributed to speciation (Wood and Guttman, 1982). Members of the complex inhabiting at least six host plant species in North America are now completely isolated by a combination of allochrony, host plant preferences, mating preferences and adaptation to their hosts. However, Wood and Guttman suggest that the initial isolation, following a rare successful host shift, was due to the effect of host phenology on egg hatch. Coupled with very low vagility this could have been sufficient to allow the population on the new host to diverge, effectively in allopatry. On the other hand, if gene flow did occur after the initial colonisation the process described here could have contributed to further divergence in emergence time.

The current difference on the most distinct hosts is about 7 days for hatch time and about 10 days for adult emergence. However, there is a considerable delay between emergence of adults and sexual maturity, as indicated by the appearance of precopulatory pairs, and the latter is more nearly equivalent to "emergence time" in my model. The difference in maturation time is about 25 days, with a standard deviation of 7 to 9 days within host associated populations. The heritability is unknown. Survival is limited, especially in males for which the average time of death is only about 5 days after the average time of appearance of precopulatory pairs. Vagility is very low so that most females lay eggs on their own host species, most frequently on the individual on which they emerged. Male vagility is greater than in females but is also low. Clearly those parameters that have been measured fall within the range used in the model and so the potential for differential gene flow to influence divergence does exist. There is now very little overlap in maturation time but the overlap may have been greater in the past and, in any case, the opportunity for gene flow is also influenced by the survival of males and females. It may be that the greater difference in timing of maturation than of hatch or adult emergence reflects divergence by this mechanism, although alternative explanations certainly exist including variation in the nutritional quality of the hosts.

Differential gene flow deserves wider consideration in the context of host associated divergence of phytophagous insects as it does for closely adjacent plant populations. No doubt it has the potential to influence other characters in other settings as well.

Acknowledgements I am grateful to Richard Nichols and Paul Brakefield for helpful discussions and to the Royal Society for financial support.

\section{REFERENCES}

BUSH, G. L. 1975. Modes of animal speciation. Ann. Rev. Ecol. Syst., 6, 339-364.

BULMER, M. G. 1980. The Mathematical Theory of Quantitative Genetics. Oxford University Press, Oxford.

BUTLIN, R. K. 1989. Reinforcement of premating isolation. In Otte, D. and Endler, J. A. (eds) Speciation and its Consequences, Sinauer Assoc., Sunderland, Mass.

DIEHL, S. R. AND BUSH, G. L. 1989. The role of habitat preference in adaptation and speciation. In Otte, D. and Endler, J. A. (eds) Speciation and its consequences, Sinauer Assoc., Sunderland, Mass.

McNEILLY, T. AND ANTONOVICS, J. 1968. Evolution in closely adjacent plant populations. IV. Barriers to gene flow. Heredity, 23, 205-218.

PATERSON, H. E. H. 1985. The recognition concept of species. In Vrba, E. S. (ed.) Species and Speciation, Transvaal Museum, Pretoria.

SMITH, D. C. 1988. Heritable divergence of Rhagoletis pomonella host races by seasonal asynchrony. Nature, 336, 66-67.

STAM, P. 1983. The evolution of reproductive isolation in closely adjacent plant populations through differential flowering time. Heredity, 50, 105-118.

THODAY, J. M. AND GIBSON, J. B. 1970. Environmental and genetical contributions to class difference: a model experiment. Science, 167, 990-992.

WOOD, T. K. AND GUTTMAN, S. I. 1982. The ecological and behavioral basis for reproductive isolation in the sympatric Enchenopa binotata complex (Homoptera: Membracidae). Evolution, 36, 233-242. 